\section{Is low blood cholesterol level associated with increased risk of hemorrhagic stroke?}

The risk of ischemic stroke grows with increasing blood cholesterol level. It has been suggested, however, that lowering blood cholesterol level might increase the risk of hemorrhagic stroke. Ebrahim et al. have investigated the association of blood cholesterol level with stroke subtype in a cohort of 787,442 Korean civil servants (84\% male).

Healthy participants were categorized to one of six groups on the basis of their cholesterol level. Between 1 August 1990 and 31 July 2001, 6,328 participants had an ischemic stroke, 3,947 had a hemorrhagic stroke, 3,170 had an undefined stroke, and 4,417 had a myocardial infarction. Risk of ischemic stroke and risk of myocardial infarction both grew with increasing serum cholesterol level (hazard ratios 1.20 and 1.48 per $1 \mathrm{mmol} / \mathrm{l}$ increase in serum cholesterol). Risk of hemorrhagic stroke decreased slightly with increasing serum cholesterol level (hazard ratio 0.91 per $1 \mathrm{mmol} / \mathrm{l}$ increase in serum cholesterol); this weak inverse association occurred only in patients with hypertension. Gamma glutamyl transferase level, which reflects alcohol consumption, was positively associated with hemorrhagic stroke risk. Increased hemorrhagic stroke risk in patients with low blood cholesterol levels occurred only in those who also had high levels of gamma glutamyl transferase.

The authors conclude that low blood cholesterol level might act as a marker of the damaging effects of alcohol, rather than being a cause of hemorrhagic stroke. They state that lowering cholesterol level to within the range likely to be achieved using statins is unlikely to increase the risk of hemorrhagic stroke.

Original article Ebrahim S et al. (2006) Serum cholesterol, haemorrhagic stroke, ischaemic stroke, and myocardial infarction: Korean national health system prospective cohort study. BMJ 333: 22

\section{ALIAS trial of high-dose human albumin for acute stroke produces encouraging results}

Promising results were recently reported for the ALIAS (Albumin in Acute Stroke) Pilot Trial-a phase I, open-label, dose-escalation study investigating use of high-dose human albumin (ALB) for the treatment of acute ischemic stroke. ALB has previously been shown to have strong neuroprotective properties in rodent models of stroke.

A total of 82 patients with acute ischemic stroke participated in the study. Six successive cohorts received an infusion of 25\% ALB within $16 \mathrm{~h}$ of stroke onset, with dose tiers increasing from $0.34 \mathrm{~g} / \mathrm{kg}$ to $2.05 \mathrm{~g} / \mathrm{kg}$. Forty-two of the patients also received intravenous tissue plasminogen activator-the current standard of care for acute ischemic stroke.

In addition to regular monitoring of vital signs, patients underwent regular cardiac evaluation and were given brain CT scans and chest X-rays after treatment. Patients were assigned NIHSS (NIH stroke scale) scores at baseline, at regular intervals during the first $72 \mathrm{~h}$ after treatment, at discharge and 1 month after discharge. At 3 months post-discharge, efficacy outcomes were determined using the NIHSS scale, modified Rankin scale ( $m R S)$ and Barthel Index.

After adjustment for the effects of tissue plasminogen activator, the probability of a good outcome at 3 months (defined as an NIHSS score of $0-1$ and/or an mRS score of $0-1$ ) for the three highest ALB doses was found to be $81 \%$ higher than for the three lowest doses, and $95 \%$ higher than for comparable patients from the NINDS rt-PA Stroke Study. The only adverse event was mild to moderate pulmonary edema, which occurred in $13 \%$ of subjects and was successfully managed with diuretics.

On the basis of these results, a large phase III trial is now underway, and is expected to conclude in 2010.

Original articles Ginsberg MD et al. (2006) The ALIAS Pilot Trial: a dose-escalation and safety study of albumin therapy for acute ischemic stroke-I: physiological responses and safety results. Stroke 37:2100-2106

Palesch YY et al. (2006) The ALIAS Pilot Trial: a doseescalation and safety study of albumin therapy for acute ischemic stroke-II: neurologic outcome and efficacy analysis. Stroke 37: 2107-2114

\section{Interferon- $\beta$ therapy for multiple sclerosis is linked with headache}

Studies indicate that the frequency of headaches in patients with multiple sclerosis (MS) is significantly higher in those receiving 